ASTHMA

\title{
Negative life events and quality of life in adults with asthma
}

\author{
C Archea, I H Yen, H Chen, M D Eisner, P P Katz, U Masharani, E H Yelin, G Earnest, P D Blanc
}

Thorax 2007;62:139-146. doi: 10.1136/thx.2006.065730

See end of article for authors' affiliations

......................

Correspondence to:

Dr P D Blanc, Division of

Occupational and

Environmental Medicine,

Department of Medicine,

University of California San

Francisco, 350 Parnassus

Avenue, Suite 609, San

Francisco, CA 94117, USA;

paul.blanc@ucsf.edu

Received 17 May 2006

Accepted 2 August 2006

Published Online First

23 August 2006

\begin{abstract}
Background: The relationship between stress and quality of life in adults with asthma has not been well studied. Stress, quantified by negative life events, may be linked to quality of life in asthma through multiple pathways, including increase in disease severity and adverse effects on socioeconomic status (SES).

Methods: The responses to a self-completed questionnaire assessing negative life events (NLEs) in the previous 12 months (from a 24-item checklist) among 189 adults with asthma from a well-characterised cohort were analysed. The relationship between the number of NLEs reported and asthma-specific quality of life (AQOL) was measured with the Marks instrument. General linear modelling was used to test the conjoint effects of NLEs, SES and disease severity based on the Severity of Asthma Score, a validated acute and chronic disease measure.

Results: Those with annual family incomes $<\$ 60000$ reported significantly more NLEs than those with higher incomes $(p=0.03)$. The number of NLEs did not differ significantly between those with forced expiratory volume in $1 \mathrm{~s}<80 \%$ predicted and those with $>80 \%$ predicted, nor among those with lower compared with higher Severity of Asthma Score. The frequency of NLEs was associated with poorer (higher numerical score) AQOL $(p=0.002)$. When studied together in the same model, combinations of income level and asthma severity (greater or lesser Severity of Asthma Score; $p<0.001)$ and number of NLEs $(p=0.03)$ were both significantly associated with $A Q O L$.

Conclusion: NLEs are associated with quality of life among adults with asthma, especially among those of lower SES. Clinicians should be aware of this relationship, especially in vulnerable patient subsets.
\end{abstract}

$\mathrm{T}$ he relationship between stress and quality of life in adults with asthma is believed to be important, but has not been well studied. In theory, stress may affect the quality of life in asthma through multiple different mechanisms, including mediation via a step-up in disease severity or through psychosocial pathways that may modulate the impact of disease and how it is perceived at the individual level, either of which may affect the quality of life. ${ }^{12}$ Quality of life in asthma is increasingly recognised as a clinically important health measure, reflecting the effect of disease from the patient's perspective..$^{3-5}$

There is no single approach to assessing stress in relation to health, adding further challenges to the systematic study of this problem. One method of quantifying stress that has been applied in a number of different contexts assesses recent "major life events". This approach focuses on distinct experiences that may have occurred over a discrete period of time. These are ascertained through various survey tools, ranging from semi-structured interviews to a self-completed checklist. Although such life events were originally conceived as being either positive or negative, but most importantly engendering "social readjustment", the construct has evolved to emphasise "stressful life events", focusing in particular on experiences generally considered to be negative stressors. ${ }^{6-9}$ Examples of negative stressors include death of a partner, family member or close friend, major financial loss, and experience of a major personal crime or injury.

We wished to test whether negative life events were associated with poorer asthma-specific quality of life among adults with asthma, consistent with an adverse effect of stress on health. For this analysis, we used data from a wellcharacterised asthma cohort, taking into account asthma severity and personal demographics insofar as they might confound any observed effects of negative life events.

\section{METHODS}

\section{Overview}

In this cohort study of adults with asthma, we analysed responses to a self-completed questionnaire assessing life events in relation to other survey data and measured lung function. The patients studied represent a subset of a larger cohort of adults with asthma and chronic rhinitis followed longitudinally as part of an ongoing observational study. ${ }^{10}$ The patients completed a life events questionnaire during a home visit at which their lung function was also assessed. ${ }^{11}$ Before the home visit itself, extensive survey data were gathered through a comprehensive structured telephone interview. This analysis focuses on the inter-relationships among asthma severity (based on telephone interview data and lung function tested at the home visit), asthma-specific quality of life (derived from a battery included in the telephone interview) and the results of the life events questionnaire (completed during the home visit).

\section{Patient recruitment and follow-up}

The study cohort is derived from an original recruitment of patients with asthma based on a random sampling of pulmonary and allergy specialists in Northern California, followed by a subsequent recruitment from family practice specialists. ${ }^{12}{ }^{13}$ Later, a third group of patients, also in Northern California, was recruited through random digit dialling. ${ }^{14}$ This final recruitment wave included people with a self-reported physician diagnosis of asthma, rhinitis or both conditions. Beginning in 2000-1, these patients were integrated into a single ongoing cohort completing the same structured telephone interview and followed regularly thereafter.

Figure 1 summarises the patient recruitment and retention. The combined cohort $(\mathrm{n}=548)$ was interviewed together for the first time in 2000-1. Of these patients, 102 had rhinitis alone without asthma and are not considered further in this analysis. In the follow-up, carried out in 2002-3, we successfully re-interviewed 347 of $446(81 \%)$ patients with asthma. Of

Abbreviations: ALEQ, Asthma Life Events Questionnaire; AQOL, Asthma Quality of Life; $\mathrm{FEV}_{1}$, forced expiratory volume in $1 \mathrm{~s}$; MCS, Mental Component Summary; NLE, negative life event; PCS, Physical Component Summary; SES, socioeconomic status; SF-36, short form health survey 36 


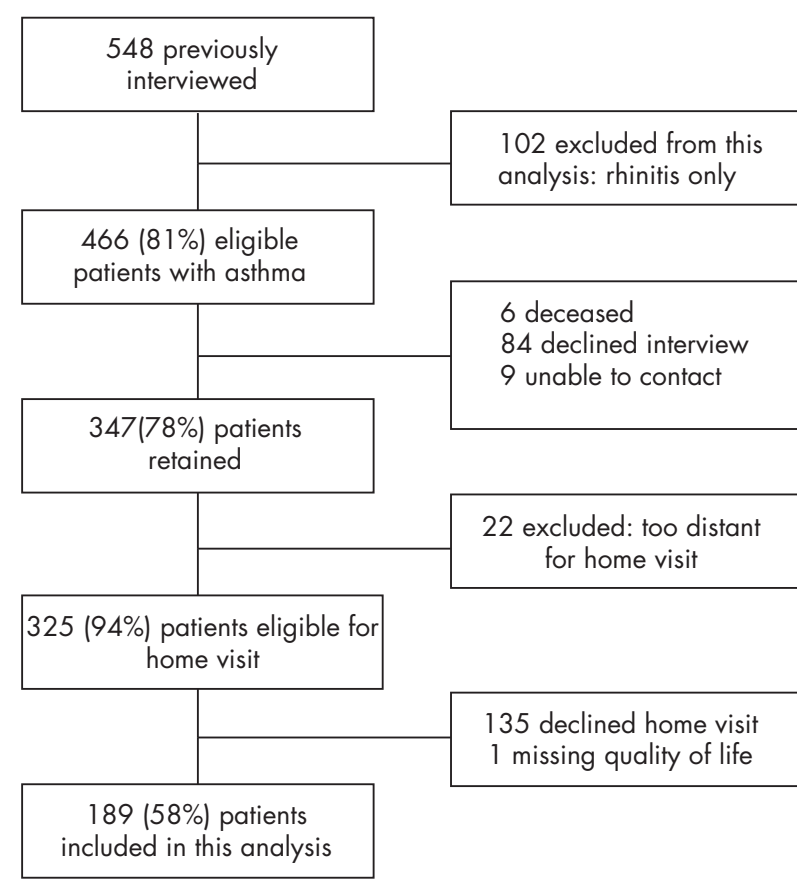

Figure 1 Patient inclusion and retention in follow-up studies used in this analysis.

those not re-interviewed, 6 (1\%) patients had died, 84 (19\%) declined participation and $9(2 \%)$ could not be successfully contacted. Details of follow-up and analyses of the complete dataset derived from these interviews, including patients with rhinitis alone, have been reported previously. ${ }^{10}{ }^{11} 15-19$

\section{Structured telephone interview content}

Data collection was performed using a structured interview averaging $45 \mathrm{~min}$ in duration. The survey instrument included questions covering asthma severity (medical history, symptoms and drugs), an asthma-specific quality of life instrument, and survey items dealing with demographics and socioeconomic status (SES).

\section{Home visits}

After the telephone interviews, patients were invited to participate in the home visit component of the study. The average time elapsed between the telephone interview and the home visit was 11 weeks. The home visit protocol included multiple components, including environmental and biological sampling protocols that have been reported previously. ${ }^{11}{ }^{17}$ Of the 325 patients with asthma living in northern California and thus geographically eligible for this component of the study, $190(58 \%)$ underwent a home visit. We excluded one of these patients from analysis due to missing quality of life data, leaving a final study group of 189 for this study. We found no significant differences ( $p>0.20$ in all cases) in age, sex, race and ethnicity, age, education, or smoking status between 189 patients with asthma from the cohort who were included in this analysis and the 136 who were not.

Adaptation of a life events questionnaire for this study The life events questionnaire we gave was adapted from the Life Experiences Survey, which in turn is based on the Social Readjustment Rating Scale. ${ }^{6}{ }^{8}$ The life events questionnaire was completed only at the time of the home visit; it was not included in the telephone survey. Consistent with the recommendations of Turner and Wheaton, we tailored our checklist so that it was consistent with the target population, retained a core set of events (consistent with life event checklists commonly used), held to a target range of 30-50 events queried, and specified a 12-month time frame for the events reported. ${ }^{12}$ The checklist adaptation we administered was comprised of 40 closed-ended items and two additional optional open-ended items where additional stressors could be listed by the respondent. Examples include major illness or death of a family member or close friend, purchasing a home, or starting a new business. For each item identified, patients were asked to provide a stress weighting between 0 and 10 , yielding a maximum possible score of 420 (including the two open-ended events). Our questionnaire differed from the format of the original Life Experiences Survey in several key ways.

1. We did not include items worded separately by sex or marital status or for students.

2. We used language neutral to sexual orientation (eg, spouse/partner).

3. We added items related to physical assault, robbery, motor vehicle accident and death of a pet that were not in the original checklist.

4. We expanded the number of checklist items concerning personal finances and working conditions.

5. The scoring system we used to assess event-specific stress did not require that respondents specify a "positive" or "negative" value to their experience.

Table 1 details the battery that we administered, which we will refer to as the Asthma Life Events Questionnaire (ALEQ). Consistent with the structure of the original Life Experiences Survey, some of the life experiences are inherently negative stressors, whereas some items query about events that may be stressful but are not necessarily negative. Therefore, we limited this analysis to the 26 items from the complete ALEQ that more likely than not reflect negative events. For example, we included in the analysis death and loss (overwhelmingly negative), the end of a long-term relationship (nearly always a negative event) and someone moving out of the home (potentially negative or positive, but in balance more likely to have negative aspects). By contrast, we excluded starting a new job because, although this can be a major stressor, it is inherently neither positive nor negative and indeed may be more likely to be the former than the latter. This classification is consistent with the generally accepted analytical approaches to major life experiences that have shown the numerical count of negative events to be the most predictive of health outcomes, without added explanatory power from additional weighting (eg, by perceived stress). ${ }^{20}{ }^{21}$ In addition to items ascertaining events that were not clearly negative, we also excluded from the analysis two items related to illness and hospitalisation. We did this because of their obvious link to the health outcome measures of interest. This exclusion is also consistent with standard approaches to analysing life events in relation to health outcomes..$^{20}$ After all exclusions, we retained 24 closedended items for this analysis from the total ALEQ. We also analysed open-ended responses, limited to those that were clearly negative events.

\section{Disease severity, asthma-specific quality of life and general health status}

These measures were based on data obtained through the structured telephone interviews. Asthma severity was quantified using the Severity of Asthma Score, a previously validated battery that is a composite of current asthma symptoms, past asthma hospital admissions, current and past systemic corticosteroid use, recent use of other asthma drugs and lifetime experience of certain types of healthcare use. ${ }^{22}{ }^{23}$ A maximum 
Table 1 The 24 major life event items analysed among 189 respondents

\begin{tabular}{|c|c|c|}
\hline \multirow[b]{2}{*}{ Item } & Content & \multirow{2}{*}{$\begin{array}{l}\text { Frequency } \\
\text { n }(\%)\end{array}$} \\
\hline & Did this event occur during the past 12 months? & \\
\hline 1. & Death of a spouse or partner & $4(2)$ \\
\hline 2. & You cared for a seriously ill or disabled family member & $62(33)$ \\
\hline 3. & Someone close to you died (other than spouse or partner) & $46(24)$ \\
\hline 4. & Your pet died & $39(21)$ \\
\hline 5. & You lost or had to move out of a home through fire, flood or other disaster & $2(1)$ \\
\hline 6. & Someone moved out of your home & $37(20)$ \\
\hline 7. & You (or someone close to you) experienced a physical assault or attack & $16(8)$ \\
\hline 8. & You (or someone close to you) were robbed or burglarised & $16(8)$ \\
\hline 9. & You (or someone close to you) were involved in a major car accident & $24(13)$ \\
\hline 10. & You (or someone close to you) were involved in a personal law suit or court case & $34(18)$ \\
\hline 11. & You experienced a foreclosure on a mortgage or loan & $3(2)$ \\
\hline 12. & You did not have enough money to pay bills & $49(26)$ \\
\hline 13. & You needed to provide financial assistance to someone close to you & $61(32)$ \\
\hline 14. & You did not get an expected wage or salary increase or promotion & $27(14)$ \\
\hline 15. & You (or your spouse/partner) took a cut in pay or salary & $49(26)$ \\
\hline 16. & Your (or your spouse's/partner's) work conditions changed for the worse & $57(30)$ \\
\hline 17. & Your (or your spouse's/partner's) workload increased & $87(46)$ \\
\hline 18. & You had trouble with employer or boss & $50(26)$ \\
\hline 19. & You (or your spouse/partner) were fired, laid off or became unemployed & 33 (17) \\
\hline 20. & You experienced a major increase in the number of arguments with spouse/partner & $24(13)$ \\
\hline 21. & You had a major argument with another family member or friend & 45 (24) \\
\hline 22. & Friends or family moved away & $27(14)$ \\
\hline 23. & You divorced or separated from your spouse/partner & $13(7)$ \\
\hline 24. & You ended another long-term or serious relationship & $9(5)$ \\
\hline
\end{tabular}

The following two health-related items were included in the battery, but were excluded from the analysis because they would be anticipated to be linked to asthma severity and thus to quality of life (see Methods):

1. You became seriously ill or injured (reported by $58(31 \%))$

2. You had surgery or were hospitalised (reported by $43(23 \%)$ )

The following were included in the questionnaire administered, but were excluded from this analysis because they could be either positive or negative events (see Methods)

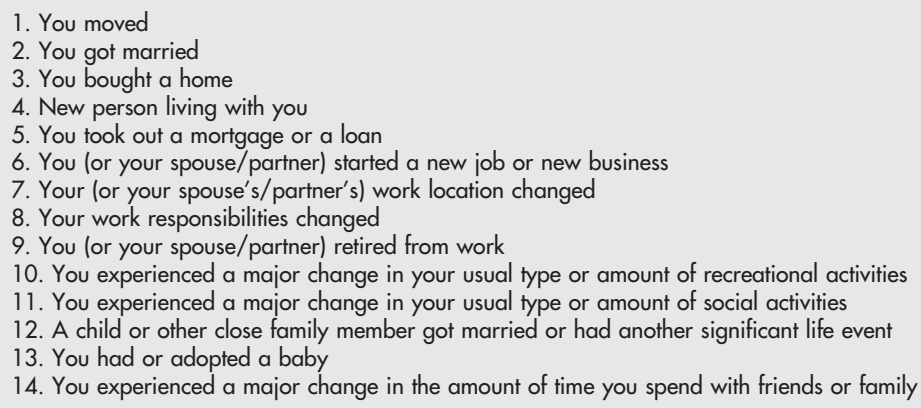

score of 28 , which is weighted heavily by treatment received, reflects greater asthma severity. We found no significant difference in scores between those included $(n=189)$ and excluded $(n=136)$ for this analysis: mean difference $0.5(95 \%$ confidence interval (CI) -0.7 to 1.7 points). We also classified patients according to the Global Initiative for Asthma guidelines using the algorithm of Laird et al. ${ }^{24}$ Inhaled steroid dosing and other anti-inflammatory drug use was based on direct inspection of drugs given to patients at the time of the home visit. The Asthma Quality of Life (AQOL) questionnaire is a validated, asthma-specific instrument using a 20 -item Likerttype scale we have adapted for telephone administration. ${ }^{25}{ }^{26}$ It assesses four domains: breathlessness, mood, social functioning and health concerns. A total score and subscale scores can be calculated, with higher scores reflecting poorer quality of life. General health status was assessed with the short form health survey 12 (SF-12) yielding both Physical Component Summary (PCS) and Mental Component Summary (MCS) scores. Normative scores are PCS, 53 (7) and MCS, 49.5 (9) among
US adults aged $18-44$ years without chronic morbidity. ${ }^{27} 28$ For those patients from the asthma cohort included $(\mathrm{n}=189)$ compared with those not included $(\mathrm{n}=136)$ in this analysis, there were no significant differences in AQOL (mean difference $0.8 ; 95 \%$ CI -2.4 to 4.1 ; data missing for one patient), SF-12 PCS (mean difference $-1.0 ; 95 \%$ CI -3.4 to 1.5 ) or SF-12 MCS (mean difference $-0.2 ; 95 \% \mathrm{CI}-2.1$ to 1.5 ).

\section{Lung function and cotinine}

At the time of the home visit, we performed spirometry using an EasyOne spirometer (ndd Medical Technologies, Chelmsford, Massachusetts, USA). The EasyOne spirometer meets ATS 1994 diagnostic standards for spirometry measurements. ${ }^{29}$ Spirometry measurements were taken using a standard protocol conforming to ATS guidelines to obtain the forced expiratory volume in $1 \mathrm{~s}\left(\mathrm{FEV}_{1}\right){ }^{30}$ We calculated the $\mathrm{FEV}_{1}$ as a percentage of the age, height and sex values using the predictive equations of Hankinson et al. ${ }^{31}$ At the time of the home visit, serum cotinine levels were obtained in never and 
Table 2 Cumulative negative life event frequency among 189 patients surveyed

\begin{tabular}{ll}
\hline & Frequency reporting \\
\cline { 2 - 2 } Number of events & $\mathbf{n}(\%)$ \\
\hline $0-2$ & $59(31)$ \\
$3-4$ & $57(30)$ \\
$5-6$ & $35(19)$ \\
$\geqslant 7$ & $38(20)$ \\
\hline
\end{tabular}

former smokers to assess second-hand smoke exposure. ${ }^{11}$ We reclassified five patients from ex-smoking (based on interview responses) to current smoking status because they had serum cotinine levels $>14 \mathrm{ng} / \mathrm{ml}$.

\section{Data analysis}

In addition to analysing the count of negative life events as a continuous variable, we also categorised the frequency using cut-points approximating quartiles. For other variables of interest, we defined strata based on cut-points approximating the median value or, in the case of $\mathrm{FEV}_{1} \%$ predicted value, the commonly accepted cut-point of $80 \%$ of predicted. The Wilcoxon's rank sum was used to test differences in the number of reported negative life events for each subject by dichotomously defined demographic variables, Severity of Asthma Score and $\mathrm{FEV}_{1}$ percentage predicted. We analysed the relationship between the number of negative life events as an independent variable and AQOL (total and subscales) as dependent variables using linear regression analysis. Further analyses for total AQOL score were stratified by income and lung function. The Severity of Asthma Score was added to these models in multiple linear regression analyses to test for mediation by asthma severity. Generalised linear analysis of variance models tested negative life events (categorised as $0-2$; $3-4 ; 5-6 ;$ or $\geqslant 7$ events) and each of four income-asthma severity combinations (categorised as low-low, low-high, highlow and high-high) as predictors of total AQOL score. We retested the same predictive models with SF-12 PCS and SF-12 MCS as the dependent variables. All analyses used a standard statistical package (SAS V.9.0).

\section{RESULTS}

Frequency of negative events reported in the ALEQ

Table 1 shows the frequencies for specific negative life events. The most frequently reported event was an increase in workload (46\% of patients). Losing or moving out of a home due to fire, flood or other disaster $(\mathrm{n}=2,1 \%)$ was the least commonly reported event. Table 2 summarises the cumulative frequency of negative events. The mean (standard deviation (SD)) number of negative life events reported overall was 4.3 (3.1; median $=4$; interquartile range 2-6; absolute range $0-17$ ). No events were reported among $14(7 \%)$ patients. In all, 50 patients reported at least one negative life event as an openended response; the most frequently cited were concerns about finances $(n=8)$ and the health of family and friends $(n=6$; data not included in table 2 ).

\section{Demographic and health status variables}

Table 3 presents the demographics and smoking status for study patients. The group was predominantly female (69\%), white, non-Hispanic (71\%) and with high educational attainment ( $49 \%$ college graduates). There were few current smokers $(\mathrm{n}=10 ; 5 \%)$. The mean (SD) age was $46(9)$ years.

The mean (SD) $\mathrm{FEV}_{1} \%$ predicted was 83 (18) among all subjects; it was $<80 \%$ in $71(38 \%),<70 \%$ in $46(24 \%)$ and
Table 3 Demographic and smoking variables among 189 adults with asthma

\begin{tabular}{|c|c|}
\hline Demographic and smoking variables & Frequency \\
\hline $\begin{array}{l}\text { Age in years, mean (SD) } \\
\text { Female sex, } \mathrm{n}(\%)\end{array}$ & $\begin{array}{r}46(8.8) \\
131(69)\end{array}$ \\
\hline $\begin{array}{l}\text { Education } \\
\text { High school or less, n (\%) } \\
\text { Some college, n (\%) } \\
\text { College graduate, n (\%) }\end{array}$ & $\begin{array}{l}32(17) \\
64(34) \\
93(49)\end{array}$ \\
\hline $\begin{array}{l}\text { Annual family income } \\
<\$ 20000, \mathrm{n}(\%) \\
\$ 20000-40000, \mathrm{n}(\%) \\
\$ 40000-80000, \mathrm{n}(\%) \\
\geqslant \$ 80000, \mathrm{n}(\%)\end{array}$ & $\begin{array}{l}19(10) \\
37(20) \\
53(28) \\
80(42)\end{array}$ \\
\hline White, non-Hispanic, n (\%) & $134(71)$ \\
\hline $\begin{array}{l}\text { Smoking status } \\
\text { Never, n (\%) } \\
\text { Ex smoker, n (\%) } \\
\text { Current smoker, n (\%) }\end{array}$ & $\begin{array}{c}124(66) \\
55(29) \\
10(5)\end{array}$ \\
\hline
\end{tabular}

$<60 \%$ in $23(12 \%)$. Classified by the Global Initiative for Asthma criteria (table 4), the patients fell into three categories of approximately similar size: steps $1-2$ (intermittent to mild asthma; 40\%); step 3 (moderate; 29\%) and step 4 (severe; $31 \%$ ).

The mean (SD) Severity of Asthma Score was 7.8 (5.2) points. It was correlated with $\mathrm{FEV}_{1} \%$ predicted (Spearman $\mathrm{r}=-0.39 ; \mathrm{p}<0.001)$ and with the Global Initiative for Asthma classification (analysis of variance $\mathrm{F}=26.2 ; \mathrm{p}<0.001$ ). The SF-12 PCS (44.7 (11.1)) and SF-12 MCS (46.8 (7.7)) were both modestly lower than the population normative mean value of 50. The asthma-specific QOL score (mean (SD) 16.1 (15.2)) correlated with the PCS (Spearman $r=-0.51 ; p<0.001$ ) in the expected direction (poorer quality of life (higher score) with poorer SF-12 PCS (lower score)). The correlation between asthma-specific QOL and SF-12 MCS, although also in the expected direction, was weaker (Spearman $\mathrm{r}=-0.16$; $\mathrm{p}=0.03)$.

Frequency of negative life events by demographics and asthma severity

We found no substantive differences in the frequency of negative life events stratified by demographic covariates except for income strata and smoking status (table 5). The number of negative life events did not differ significantly between those with $\mathrm{FEV}_{1}<80 \%$ and those with $\geqslant 80 \%$ predicted, nor among those with lower compared with higher Severity of Asthma scores.

\section{Negative life events in relation to asthma-specific quality of life}

We used linear regression analysis to test whether an increase in negative life events was associated with poorer asthmaspecific quality of life (reflected in higher AQOL scores). As table 6 shows, with each additional negative life event, the total AQOL score increased (a decrement in quality of life) by approximately 1.1 point $(\mathrm{p}=0.002)$. When stratified by income level, there was a significant association of negative life events with poorer AQOL among those with lower incomes $(\beta=1.7$, $95 \%$ CI 0.5 to $2.9 ; \mathrm{p}=0.005)$, but not among those with higher incomes $(\beta=0.3,95 \% \mathrm{CI}-0.5$ to $1.1 ; p=0.43)$. Among the subset of patients with annual family incomes of $\leqslant \$ 20000 /$ year $(n=19$; data not shown separately in table 6$)$, the slope of 


\begin{tabular}{|c|c|}
\hline Health status variables & Frequency \\
\hline $\mathrm{FEV}_{1} \%$ predicted, mean (SD) & $83.1(18.4)$ \\
\hline \multicolumn{2}{|l|}{ Global Initiative for Asthma (GINA) Severity } \\
\hline Step 1 (intermittent), n (\%) & $49(26)$ \\
\hline Step 2 (mild persistent), $\mathrm{n}(\%)$ & $26(14)$ \\
\hline Step 3 (moderate persistent), $\mathrm{n}$ (\%) & $55(29)$ \\
\hline Step 4 (severe persistent), n (\%) & $59(31)$ \\
\hline Asthma Severity Score, mean (SD) & $7.8(5.2)$ \\
\hline SF-12 Physical Component Score, mean (SD) & $44.7(11.1)$ \\
\hline SF-12 Mental Component Score, mean (SD) & $46.8(7.7)$ \\
\hline Quality of life (total score), mean (SD) & $16.1(15.2)$ \\
\hline \multicolumn{2}{|l|}{$\begin{array}{l}\text { Quality of life subscales } \\
\text { Q }\end{array}$} \\
\hline Physical impact, mean (SD) & $4.6(4.5)$ \\
\hline Emotional impact, mean (SD) & $4.2(4.2)$ \\
\hline Social impact, mean (SD) & $4.3(5.8)$ \\
\hline Health concerns, mean (SD) & $2.9(2.7)$ \\
\hline
\end{tabular}

the relationship of negative life events to poorer AQOL was even steeper $(\beta=2.8,95 \%$ CI 0.2 to $5.4 ; p=0.04$ ).

We also found differences when stratified by lung function (table 5). The number of negative life events was associated with poorer AQOL among those without airflow obstruction $\left(\mathrm{FEV}_{1}\right.$ percentage predicted $\left.>80 \%\right)(\beta=1.5,95 \%$ CI 0.7 to 2.3 ; $\mathrm{p}<0.001$ ), but there was no significant association between the number of negative events and AQOL $(p=0.26)$ among those with airflow obstruction. In addition, among smokers, the number of negative life events was significantly associated with AQOL $(\beta=1.6,95 \%$ CI 0.5 to $2.8 ; p=0.01)$, but not among non-smokers $(p=0.14)$. In contrast with these observations, there was little difference in the association of negative life events with AQOL stratified by Severity of Asthma Score below the median (1.2 (0.4), 95\% CI 0.4 to 1.9$)$ and above the median ( $1.1(0.5), 95 \%$ CI 0.1 to $2.0 ; \mathrm{p}<0.05$ in both groups).

\section{Effect of negative life events taking into account Severity of Asthma Score}

We repeated the same analyses adjusting for Severity of Asthma Score (right-hand columns, table 6). The principal findings were similar to those in the unadjusted models, although the point estimates for the effect of negative life events was reduced in all cases. For example, adjusted for asthma severity, the AQOL score changed in the direction of poorer quality of life by only 0.7 points (95\% CI 0.1 to 1.3 ) for each life event $(p=0.02)$. Reanalysing these models, including the negative events from open-ended responses, did not substantively alter the observed findings. We repeated this analysis for each of the four asthma-specific quality of life subscales. For the subscales for physical impact ( $\beta=0.22,95 \%$ CI 0.05 to $0.39 ; p=0.01)$, emotional impact $(\beta=0.24,95 \%$ CI 0.06 to $0.41 ; p=0.01)$ and social impact $(\beta=0.19,95 \%$ CI -0.05 to $0.42 ; \mathrm{p}=0.12$ ), linear regression analyses adjusted for Severity of Asthma Score yielded similar estimates of the relationship between negative life events and AQOL (although not significant for social impact). For the health concerns subscale, however, there was a lower estimated slope $(\beta=0.05$, $95 \%$ CI -0.06 to $0.15 ; \mathrm{p}=0.38$ ).

To evaluate the impact of demographic and smoking variables as potential confounders, we carried out a multiple linear regression analysis of AQOL score adding the following to negative life events and Severity of Asthma Score: sex, age,

Table 5 Frequency of negative life events: demographic and health status covariates

\begin{tabular}{|c|c|c|c|c|}
\hline \multirow[b]{2}{*}{ Demographic group } & \multirow[b]{2}{*}{ Study $\mathbf{n}$} & \multicolumn{2}{|c|}{ Frequency of negative life events } & \multirow[b]{2}{*}{$\mathrm{p}$ Value } \\
\hline & & Mean (SD) & Median (IQR) & \\
\hline $\begin{array}{l}\text { All subjects } \\
\text { Sex }\end{array}$ & 189 & $4.3(3.1)$ & $4(2-6)$ & $\begin{array}{l}\text { NA } \\
0.90\end{array}$ \\
\hline Male & 58 & $4.4(3.3)$ & $4(2-5)$ & \\
\hline Female & 131 & $4.2(3.0)$ & $4(2-6)$ & \\
\hline Race/ethnicity & & & & 0.35 \\
\hline White, non-Hispanic & 134 & $4.2(3.1)$ & $4(2-5)$ & \\
\hline All other & 55 & 4. $6(3.0)$ & $4(2-7)$ & \\
\hline Education & & & & 0.35 \\
\hline Some college or less & 96 & $4.5(3.2)$ & $4(2-7)$ & \\
\hline College graduate or higher & 93 & $4.1(3.0)$ & $4(2-5)$ & \\
\hline Age (years) & & & & 0.41 \\
\hline$<47$ & 90 & $4.4(2.7)$ & $4(2-6)$ & \\
\hline$\geq 47$ & 99 & $4.3(3.4)$ & $4(2-6)$ & \\
\hline Annual household income & & & & 0.03 \\
\hline$<\$ 60000$ & 82 & $4.8(3.2)$ & $4(3-7)$ & \\
\hline$\geqslant \$ 60000$ & 107 & $3.9(2.9)$ & $3(2-5)$ & \\
\hline Cigarette smoking & & & & 0.01 \\
\hline Never & 124 & $3.9(2.8)$ & $3(2-5)$ & \\
\hline Ever & 65 & $5.1(3.4)$ & $4(3-7)$ & \\
\hline $\mathrm{FEV}_{1} \%$ predicted & & & & 0.71 \\
\hline$<80$ & 71 & $4.4(3.6)$ & $4(2-6)$ & \\
\hline$\geqslant 80$ & 118 & $4.3(2.8)$ & $4(2-6)$ & \\
\hline Asthma Severity Score & & & & 0.51 \\
\hline$<7$ & 95 & $4.3(2.7)$ & $4(2-6)$ & \\
\hline$\geqslant 7$ & 94 & $4.3(3.4)$ & $4(2-6)$ & \\
\hline
\end{tabular}

$\mathrm{FEV}_{1}$, forced expiratory volume in $1 \mathrm{~s}$; IQR, interquartile range (25th-75th centile). $\mathrm{p}$ Values for all comparisons of life event frequencies are for the corresponding Wilcoxon's rank sum test. 


\begin{tabular}{|c|c|c|c|c|c|}
\hline \multirow[b]{3}{*}{ Subjects in analysis } & \multirow[b]{3}{*}{$\mathbf{n}$} & \multicolumn{4}{|c|}{ Number of negative life events } \\
\hline & & \multicolumn{2}{|l|}{ Unadjusted } & \multicolumn{2}{|c|}{ Adjusted for asthma severity } \\
\hline & & $\beta(95 \% \mathrm{Cl})$ & $\mathrm{p}$ Value & $\beta(95 \% \mathrm{Cl})$ & p Value \\
\hline All patients & 189 & & & & \\
\hline AQOL summary score & & $1.1(0.4$ to 1.8$)$ & 0.002 & $0.7(0.1$ to 1.3$)$ & 0.02 \\
\hline \multicolumn{6}{|c|}{ AQOL score, stratified by income } \\
\hline Higher income & 107 & $0.3(-0.5$ to 1.1$)$ & 0.43 & $0.2(-0.5$ to 0.8$)$ & 0.67 \\
\hline Lower income & 82 & $1.7(0.5$ to 2.9$)$ & 0.01 & $1.0(0.1$ to 2.0$)$ & 0.03 \\
\hline \multicolumn{6}{|c|}{ AQOL score, stratified by smoking status } \\
\hline Never smoker & 124 & $0.7(-0.2$ to 1.6$)$ & 0.14 & $0.5(-0.2$ to 1.2$)$ & 0.18 \\
\hline Ever smoker & 65 & $1.6(0.5$ to 2.8$)$ & 0.01 & $1.0(-0.04$ to 2.0$)$ & 0.06 \\
\hline \multicolumn{6}{|c|}{ AQOL score, stratified by lung function } \\
\hline $\mathrm{FEV}_{1} \geqslant 80 \%$ predicted & 118 & 1.5 (0.7 to 2.3$)$ & $<0.001$ & $1.1(0.4$ to 1.8$)$ & 0.002 \\
\hline $\mathrm{FEV}_{1}<80 \%$ predicted & 71 & $0.6(-0.5$ to 1.8$)$ & 0.26 & $0.3(-0.7$ to 1.3$)$ & 0.53 \\
\hline \multicolumn{6}{|c|}{ AQOL score, stratified by asthma severity } \\
\hline Asthma Severity Score $<7$ & 95 & $1.2(0.4$ to 1.9$)$ & 0.003 & NA & NA \\
\hline Asthma Severity Score $>7$ & 94 & $1.1(0.1$ to 2.0$)$ & 0.03 & NA & NA \\
\hline
\end{tabular}

smoking status, race and education. The adjusted model $\mathrm{R}^{2}$ with the addition of these six covariates changed minimally, from 0.37 to 0.38 ; none of the covariates was a significant independent predictor of AQOL score in this model, whereas both negative life events $(p=0.03)$ and Severity of Asthma Score $(p<0.001)$ remained significant.

\section{Combined effects of life events, income level and asthma severity}

To address the relationships among income, disease severity and negative life events, we tested together in a single model, combinations of income-asthma severity and negative life events. To accomplish this, we divided the study group into 16 possible cells based on income, asthma severity and ordinal category of negative life events. Patient numbers per cell ranged from 7 to 24 (median $=10)$. Figure 2 displays the mean asthma specific quality of life per cell. Patients with the combination of low income-high asthma severity had the highest AQOL scores (consistent with poorer quality of life) within each frequency level of negative life events. On analysis by generalised linear modelling, the overall model testing the relationships shown in fig 2 was found to be significant $(\mathrm{p}<0.001)$. In this model, the contribution of negative life events by ordinal category $(p=0.03)$ and income-severity category $(p<0.001)$ were both significant. Adding smoking status to the model did not substantively change these estimates, and smoking was unrelated to AQOL $(\mathrm{F}=0.08 ; \mathrm{p}=0.78)$. We also analysed the performance of the same predictive model for general health status (SF-12) as opposed to AQOL. On analysing physical health (PCS) as the dependent variable, the overall model was found to be significant $(\mathrm{p}<0.001)$, as was the contribution of income-severity group $(p=0.001)$, whereas negative life events were not significant $(p=0.10)$. For mental health (MCS), the overall model was also significant $(p=0.03)$, but neither income-severity nor negative life events were significant in the combined model ( $\mathrm{p}=0.11$ for each).

\section{DISCUSSION}

Our findings support the hypothesis that negative life events are associated with quality of life among adults with asthma. This association was observed in a complex set of relationships involving SES and asthma severity. Those in the lower income stratum reported more negative life experiences in the prior 12 months, and it was within that stratum that the number of such events was associated with poorer asthma-specific quality of life. Those with more severe asthma did not report a greater frequency of negative life events and, in multivariate modelling, asthma severity explained part, but not all of the association of such events with quality of life.

The integration of these contrasting effects yields a relationship that is shown graphically in fig 2 . Persons with severe asthma and minimal recent negative life events reported poorer quality of life across income levels. A higher frequency of negative life events, however, was associated with a decrement in asthma-specific quality of life. This was manifest as fewer negative life events within the lower income stratum, but quality of life was still negatively associated among those with higher incomes given a sufficient burden of negative life events. Among those with the greatest number of negative life events, if combined with lower income, the negative association with quality of life was present across the strata of asthma severity.

We were careful to exclude from this analysis negative life events directly related to personal health status, but we cannot disentangle fully all the potential cause and effect relationships involved. Either negative events or severity of asthma could lead directly to lower income. Indeed, a key set of the negative events we queried have explicit negative financial effects, although others (such as loss of a spouse) certainly could be fiscally as well as emotionally detrimental. Thus, we cannot say whether lower income acts as a mediator of the relationship between negative life events and quality of life or is simply a marker of negative events that also have financial effects. Our and other analyses have shown that asthma is associated with loss of work, decreased work effectiveness, change in working conditions and loss of pay..$^{12}{ }^{14-34}$ Theoretically, this could lead to more reports of selected negative events, yet we did not observe any substantive difference in number of events stratified by either Severity of Asthma Score or FEV 1 , making this an unlikely explanation for our findings.

This study is a cross-sectional analysis. Although the assessment of Severity of Asthma Score and AQOL was assessed via telephone survey that took place before the home visit at which the life events questionnaire was administered, the median time elapsed between the two was only 8 weeks, 


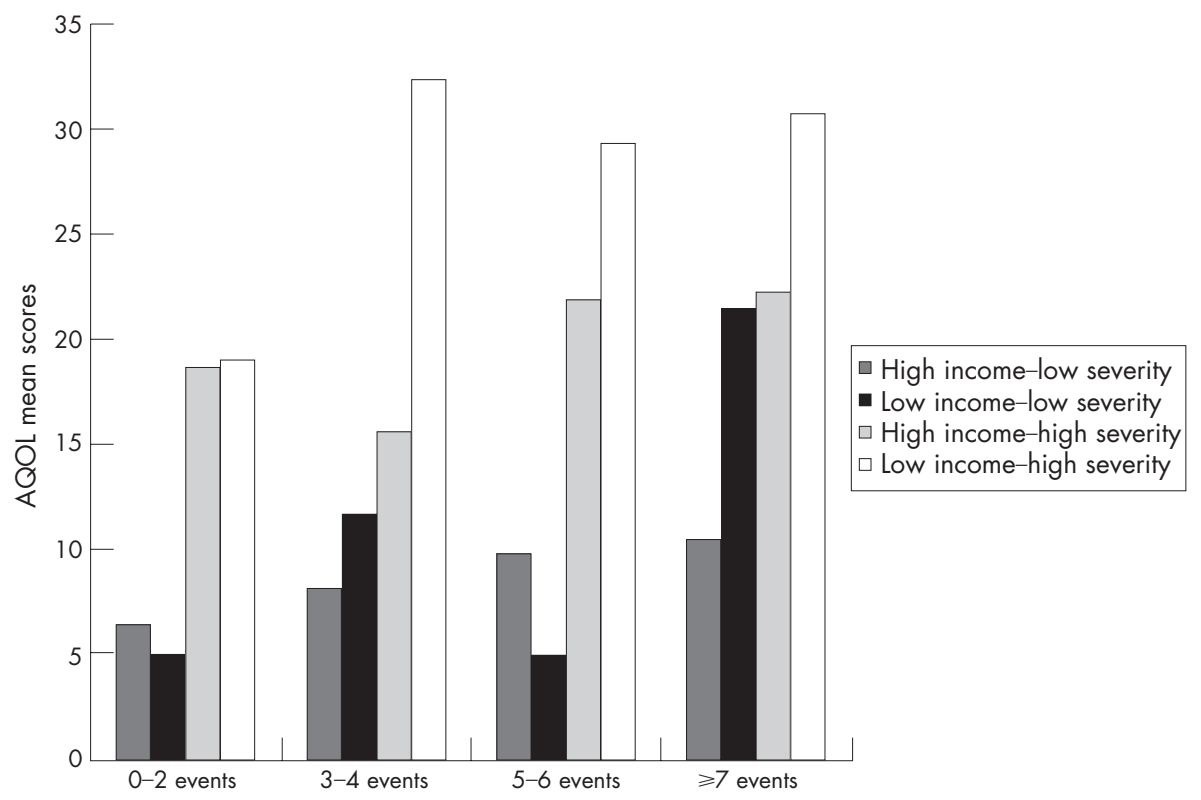

Figure 2 Association between the number of negative life events experienced in the previous 12 months and asthma-specific quality of life among 189 patients with different income-asthma severity levels. The overall model is significant $(\mathrm{F}=11.5 ; \mathrm{p}$ $<0.001$ ), as is the association for number of events $(F=3.1 ; p=0.03)$ and asthma severity-income group $(\mathrm{F}=19.0, \mathrm{p}<0.001)$. AQOL, Asthma Quality of Life.

whereas the recall period for life events was over 12 months before the home visit. We also recognise that the key study measures, by definition, are based on self-report. Thus, we cannot exclude an element of reporting bias wherein persons with certain traits-for example, anxiety-over-report negative life events and also respond to questionnaire items consistent with poorer AQOL scores. Arguing against such reporting bias as being a major factor driving our findings is the observation that the AQOL subscale "Health Concerns" was weakest in the association with reported negative life events, and yet this is the subscale that includes worry that asthma is shortening the respondent's life, worry about the future because of asthma, and fear regarding asthma control and drug dependence. ${ }^{25}$ Although recall bias could also theoretically come into play, surveys of major life events are believed to be relatively unaffected by systematic differences in reporting by disease status. ${ }^{20}$

We did not analyse the relationship between negative life events and asthma exacerbations. To the extent that increased asthma exacerbations are linked to greater disease severity, our multivariate modelling would have taken this into account and could represent "overadjustment" for this effect. A study of severe life-threatening asthma among people aged 15-49 years found no difference in the mean number of total negative life events among cases (3.9) compared with controls without asthma admitted to hospital (3.5), but a comparison group of non-hospitalised people with asthma did report significantly fewer events (2.8). ${ }^{35}$ A survey of Finnish college students reported that $21 \%$ of those with lifetime asthma reported at least one stressful life event, a significantly higher frequency than among controls (13.4\%), but exacerbations were not studied. ${ }^{36}$ A study of childhood asthma (in children aged 613 years) found that experiencing a recent severe life event was a risk factor for disease exacerbation in a 3-week period beginning 1 month after the event. ${ }^{37}$ These studies support a possible relationship between negative life events and asthma exacerbation. Exacerbation after stressful life events has been the subject of study in several chronic conditions with mixed findings; the most consistent association has been observed in multiple sclerosis. ${ }^{38}$

We did not explore in depth the potential relationships among psychological status (including depression), quality of life, life events and disease severity. We found a modest association between combined income-severity and life events as joint predictors of SF-12 mental health score (MCS). MCS was also weakly correlated with AQOL score. Analysis of more specific measures of depressive symptoms could provide additional insights into these associations. The relationship between illness severity and quality of life has been reported previously in analyses based on this cohort and other studies. ${ }^{26}{ }^{39-42}$ A recent systematic literature review on this subject underscores, however, that even the severity-quality of life relationship is complex, depending on the classification of severity used and mental health covariates in the study population. ${ }^{4}$

Limited data from other analyses of negative life events in relation to quality of life are available. An asthma-specific study including a life events battery assessed a Serbian translation of the Juniper Asthma Quality of Life instrument. ${ }^{43}$ Carried out among 160 adults with asthma in Belgrade, interviewed in 2000-2, that study reported that "stressful life events and duration of disease were not limiting factors of the quality of life of asthma patient [sic]" but did not provide data supporting this observation. Using the short form health survey 36 (SF-36) as a measure of quality of life, a study of 354 adult survivors of testicular cancer found a statistically significant association between the number of negative life events and the mental health subscale, but not the physical health subscale. This analysis took into account cancer treatment and comorbidity, as well as education (which is a common measure of SES). ${ }^{44} \mathrm{~A}$ study of 112 persons with breast cancer also found that the number of negative life events (from an abridged battery) was associated with poorer SF-36 mental health subscale scores assessed 12 months after diagnosis or surgery, taking cancerrelated stress into account, but such a relationship was not noted for physical health. ${ }^{45}$ Our secondary analysis, using the SF-12 rather than the SF-36 and taking into account income and severity, found a weak, non-significant relationship for physical health (PCS; $\mathrm{p}=0.10$ ) and mental health (MCS; $\mathrm{p}=0.11)$. The weak association we observed may be explained by the differences in measurement of quality of life using a general health status instrument compared with a diseasespecific instrument (the AQOL) and by testing a model that took into account the combined effects of income and disease severity, as well as negative life events. 
Our study suggests that negative life events are associated with a decrement in quality of life among adults with asthma, particularly among those whose baseline quality of life is relatively intact. Although wealth may not "buy" good health, better SES seems to buffer the association of negative events and health-related quality of life. Negative life events, unfortunately, may not be preventable. Nonetheless, those caring for persons with asthma should be aware that after such events, quality of life is likely to deteriorate, especially in certain subsets of patients. Among those people, attention to this potential decline and intervention, where possible, is warranted.

\section{ACKNOWLEDGEMENTS}

We thank Laura Trupin MPH for statistical review, and study personnel, including interviewers and home visit teams, for their assistance: Chito Elago, Naomi Friedling, Arleen Ervin-King, Kathryn Kern, Win Kryda, Michelle Nance, Judith Sansone, Marissa San Pedro, Karen Van der Meulen and Melanie San Pedro-Salcedo.

\section{Authors' affiliations}

C Archea, I H Yen, H Chen, M D Eisner, P P Katz, U Masharani, E H Yelin, G Earnest, P D Blanc, Department of Medicine, University of California San Francisco, San Francisco, California, USA

Funding: This work was supported by R01 ES 10906 (National Institute for Environmental Health Sciences).

Competing interests: None declared.

\section{REFERENCES}

Rietveld S, Everaerd W, Creer TL. Stress-induced asthma: a review of research and potential mechanisms. Clin Exp Allergy 2000;30:1058-66.

2 Wright RJ. Stress and atopic disorders. J Allergy Clin Immunol 2005;116:1301-6.

3 Juniper EF. Interpreting quality of life data: should we listen to the patient or the clinician? Ann Allergy Asthma Immunol 2003;91:115-16.

4 Chapman KR. Impact of 'mild' asthma on health outcomes: findings of a systematic search of the literature. Respir Med 2005:99:1350-62.

5 Ehrs P-O, Nokela M, Stälberg B, et al. Brief questionnaires for patient-reported outcomes in asthma. Validation and usefulness in a primary care setting. Chest 2006; 129:925-32.

6 Holmes TH, Rahe RH. The Social Readjustment Rating Scale. J Psychosom Res $1967 ; 11: 213-18$

7 Tennant C, Andrews G. A scale to measure the stress of life events. Aust N Z J Psychiatry 1976;10:27-32.

8 Sarason IG, Johnson JH, Siegel JM. Assessing the impact of life changes: development of the Life Experiences Survey. J Consult Clin Psychol 1978;46:932-46.

9 Paykel ES. Methodological aspects of life events research. J Psychosom Res 1983;27:341-52.

10 Blanc PD, Yen IH, Chen $\mathrm{H}$, et al. Area-level socioeconomic status and health status among adults with asthma and rhinitis. Eur Respir J 2006;27:85-94.

11 Blanc PD, Eisner MD, Katz PP, et al. Impact of the home indoor environment on adult asthma and rhinitis. J Occup Environ Med 2005:47:362-72.

12 Blanc PD, Cisternas M, Smith S, et al. Asthma, employment status, and disability among adults treated by pulmonary and allergy specialists [erratum appears in Chest 2000;1 18:564]. Chest 1996;109:688-96.

13 Blanc PD, Eisner MD, Israel L, et al. The association between occupation and asthma in general medical practice [erratum appears in Chest 2000;118:564]. Chest 1999;115:1259-64.

14 Blanc PD, Trupin L, Eisner M, et al. The work impact of asthma and rhinitis: findings from a population-based survey. J Clin Epidemiol 2001;54:610-18.

15 Yelin EH, Trupin L, Earnest G, et al. The impact of managed care on health care utilization among adults with asthma. J Asthma 2004;41:229-42.
16 Chen H, Katz PP, Eisner MD, et al. Health-related quality of life in adult rhinitis: the role of perceived control of disease. J Allergy Clin Immunol 2004; 114:845-50

17 Masharani U, Shiboski S, Eisner MD, et al. Impact of exogenous glucocorticoid use on salivary cortisol measurements among adults with asthma and rhinitis. Psychoneuroendocrinology 2005;30:744-52.

18 Chen H, Katz PP, Shiboski S, et al. Evaluating change in health-related quality of life in adult rhinitis: responsiveness of the Rhinosinusitis Disability Index. Health Quality Life Outcomes 2005;3:68.

19 Yen IH, Yelin EH, Katz P, et al. Perceived neighborhood problems and quality of life, physical functioning, and depressive symptoms. Am J Public Health 2006;96:873-9.

20 Turner RJ, Wheaton B. Checklist measurement of stressful life events. In: Cohen S, Kessler RC, Gordon LU, eds. Measuring stress. A guide for health and social scientists. New York: Oxford University Press, 1997:29-58.

21 Zuckerman MA, Oliver JM, Hollingsworth HH, et al. The comparison of life events scoring methods as predictors of psychological symptomatology. J Hum Stress 1986;12:64-70.

22 Blanc PD, Jones $M$, Besson $C$, et al. Work disability among adults with asthma. Chest 1993:104:1371-7.

23 Eisner MD, Katz PP, Yelin EH, et al. Assessment of asthma severity in adults with asthma treated by family practitioners, allergists, and pulmonologists [Erratum appears in Med Care 2000;38:880-5]. Med Care 1998;36:1567-77.

24 Liard R, Leynaert B, Zureik M, et al. Using the Global Initiative for Asthma guidelines to assess asthma severity in populations. Eur Respir $J$ 2000;16:615-20

25 Marks, GB, Dunn SM, Woolcock AJ. A scale for the measurement of quality of life in asthma. J Clin Epidemiol 1992;45:461-72.

26 Katz PP, Eisner MD, Henke J, et al. The Marks asthma quality of life questionnaire: further validation and examination of responsiveness to change [Erratum appears in J Clin Epidemiol 2001;54:105-7]. J Clin Epidemiol 1999;52:667-75

27 Ware JY, Kosiniski M, Keller SD. How to score the SF-12 health survey, 2nd edn. Boston, MA: The Health Institute, New England Medical Center 1995.

28 Gandek B, Ware JE, Aaronson NK, et al. Cross-validation of item selection and scoring for the SF-12 health survey in nine countries. J Clin Epidemiol 1998:51:1171-8.

29 Mortimer KM, Fallot A, Balmes JR, et al. Evaluating the use of a portable spirometer in a study of pediatric asthma. Chest 2003;123:1899-907.

30 American Thoracic Society. Standardization of spirometry. Am J Respir Crit Care Med 1994; 152:1107-36.

31 Hankinson JL, Odencrantz JR, Faden KB. Spirometric reference values from a sample of the general U.S. population. Am J Respir Crit Care Med 1999:159:179-87.

32 Eisner MD, Yelin EH, Katz PP, et al. Risk factors for work disability in severe adult asthma. Am J Med 2006;119:884-91.

33 Balder B, Lindholm NB, Löwhagen $\mathrm{O}$, et al. Predictors of self-assessed work ability among subjects with recent-onset asthma. Respir Med 1998;92:729-34.

34 Mancuso CA, Rincon M, Charlson ME. Adverse work outcomes and events attributed to asthma. Am J Ind Med 2003;44:236-45.

35 Kolbe J, Fergusson W, Vamos M, et al. Case-control study of severe life threatening asthma (SLTA) in adults: psychological factors. Thorax 2002:57:317-22.

36 Kilpeläinen $M$, Koskenvuo $M$, Helenius $H$, et al. Stressful life events promote the manifestation of asthma and atopic diseases. Clin Exp Allergy 2002;32:256-63.

37 Sandberg S, Järvenpää S, Penttinen A, et al. Asthma exacerbations in children immediately following stressful life events: a Cox's hierarchical regression. Thorax 2004:59:1046-51.

38 Mohr DC, Hart SL, Julian L, et al. Association between stressful life events and exacerbation in multiple sclerosis: a meta-analysis. BMJ 2004;328:731.

39 Katz PP, Yelin EH, Eisner MD, et al. Perceived control of asthma and quality of life among adults with asthma. Ann Allergy Asthma Immunol 2002:89:251-8.

40 Schatz M, Mosen D, Apter AJ, et al. Relationships among quality of life, severity, and control measures in asthma: an evaluation using factor analysis. J Allergy Clin Immunol 2005; 115:1049.

41 Horak E, Sawyer SM, Roberts M, et al. Impact of disease severity on quality of life in adults with asthma. Wien Klin Wochenschr 2005;117:462-7.

42 Eisner MD, Boland M, Tolstykh I, et al. Intensive care unit admission for asthma: a marker of severe disease. J Asthma 2005;42:315-23.

43 Spirić VT, Bogić M, Janković S, et al. Assessment of the Asthma Quality of Life Questionnaire (AQLQ): Serbian translation. Croat Med J 2004:45:188-94.

44 Fleer J, Hoekstra HJ, Sleijfer DT, et al. Quality of life of testicular cancer survivors and the relationship with sociodemographics, cancer-related variables, and life events. Support Care Cancer 2006; 14:251-9.

45 Golden-Kreutz DM, Thorton LM, Wells-Di Gregorio S, et al. Traumatic stress, perceived global stress, and life events: prospectively predicting quality of life in breast cancer patients. Health Psychol 2005;24:288-96. 